\title{
The Investigation on Rheology and Microstructure for Lithium
}

\section{Complex Grease}

\author{
Mao Jingjing ${ }^{1 *}$, Li Jianming ${ }^{1}$, Qiu Jianwei ${ }^{1}$, Liu Lijun ${ }^{1}$, Wang Yue ${ }^{1} \&$ Zhang Lijiuan $^{1}$ \\ ${ }^{1}$ Petrochina Lanzhou Lubricating Oil R\&D Institute, Lanzhou, China \\ *Mao Jingjing, E-mail: maojingjing_rhy@petrochina.com.cn
}

Received: July 25, 2019 Accepted: August 20, 2019 Online Published: September 4, 2019

doi:10.22158/ees.v2n2p54 URL: http://dx.doi.org/10.22158/ees.v2n2p54

\begin{abstract}
The rheological characteristic parameters of five lithium complex lubricating greases were determined. The comparison of rheological characteristic parameters and microstructure of the five greases was made. The results showed that the knowledge of the rheological properties of lubricating greases may contribute to reflect the change of the thickener structure. The flow transition index characterized the breaking behavior of inner structure of grease, the greater this index, the better that soap fiber structure of grease; The damping factor was shifted from a medium range towards a lower value which resulted in brittle character of sample. At a constant shear rate, the soap fiber structure of complex lithium grease is dense and uniform, apparent viscosity decline rate is small. In this sense, it is relevant to understand how the development of the soap fiber structures in the grease contributes to several functional and rheological properties of lubricating greases.
\end{abstract}

\section{Keywords}

lithium complex grease, rheological property, viscoelasticity, microstructure

\section{Introduction}

The rheology of grease refers to the flow and deformation characteristics of grease when subjected to shear stress. Grease is a colloidal structure with significant non-Newtonian fluid properties. It can maintain a certain shape like a solid when it is not subjected to shear stress, that is, it will not flow when it is at rest. When subjected to weak shear stress, it will be elastically deformed; After removing shear stress, it can return to its original position and shape, exhibiting the elastic properties of the solid. When the applied shear stress is large enough, the grease deforms and flows, and thus can no longer automatically return to the original position and shape, so the starting torque of the grease in the machinery is larger than that of the liquid lubricating oil. During the flow of the grease, as the shear stress increases, the soap fibers are aligned, which reduces the apparent viscosity of grease. In the case of extremely high shear stress, the grease flows like a Newtonian fluid, and the 
viscosity remains constant and does not change with the shear rate. The rheological properties of the grease are directly related to the microstructure of the system and the interaction between the internal materials, and are the macroscopic representation of the internal microstructure of the system. Therefore, studying the rheology of grease is of great significance for the production and application of grease.

In recent years, more and more researchers have used different methods to study the rheological properties of grease. Meng et al. studied the rheological properties of lithium grease based on thixotropy at different shear rates, different time and different temperatures. The rheological model with structural parameters was fitted from the experimental results. The model can describe the yield stress, shear dilution and thixotropy of the tested grease. Guo et al. studied the rheological properties of semi-fluid lithium grease and complex lithium grease at different consistency and temperature, and discussed the effects of the type, content, temperature and shear rate of thickeners on their rheological properties. Wang et al. studied the effects of structure and content of thickener, temperature, base oil viscosity and shear time on rheological parameters were investigated by HAAK rheometer. The results show that the strength limit of grease decreases with the increase of temperature, but the change of plastic viscosity and plastic index is irregular. Delgado et al. studied the relationship between rheological properties and microstructures in the preparation of lithium grease. J.M. Franco et al. studied the mixed rheological properties in the production of grease. J.E. Martin-Alfonso et al. studied the effect of a polymer on the rheological properties of greases. Yeong et al. studied the steady flow and viscoelasticity of greases with different thickener content. However, the test instruments and methods used by researchers are different, and the rheological performance test results are also described in different ways. The research contents are mainly focused on thixotropy, yield stress and apparent viscosity of lubricating grease rheology. The viscoelastic characteristic parameters of lubricating grease and their relationship with microstructure have not been reported.

In 2007 and 2011, the German standard revised and published the DIN 51810-1 "Determination of the shear viscosity of greases using a rotational viscometer-Part 1: Cone and plate inspection systems", DIN 51810-2 “Determination of lubrication Rheological properties-Part 2: Determination of the flow point using an oscillating rheometer with a parallel plate detection system. The standard specifies the test methods and conditions for grease rheology, but the standard is not used to describe how the test results should be understood and applied. In addition, therefore, this paper applies and discusses the standard method through literature and data accumulation. The rheological tests of several lithium complex grease samples were carried out, and the test results were analyzed. The microstructure of the samples was compared with the samples. It is believed that by testing the rheological parameters of lubricating grease, it has certain guiding significance for the development and application of grease products. 


\section{Experiment Part}

\subsection{Test Equipment}

The electron micrograph was taken by a field emission scanning electron microscope (FE-SEM) manufactured by JEOL, model number JSM-6701F. The rheology test uses Anton Paar's MCR301 rheometer with air bearings, motors and optical encoders. It can be extended to different temperature control units with control stress and control rate functions. The oscillating mode experiment used a parallel plate test system with a diameter of $25 \mathrm{~mm}$. The rotation mode experiment used a cone and plate test system with a diameter of $25 \mathrm{~mm}$ and an angle of $1.0^{\circ} \mathrm{C}$. Test temperature accuracy \pm 0.1 ${ }^{\circ} \mathrm{C}$.

\subsection{Test Methods}

Refer to DIN 51810-1 and DIN 51810-2.

\subsection{Preparation and Performance of Grease Samples}

The base oil and 12-hydroxystearic acid were added to the kettle and stirred, and the mixture was heated to $80{ }^{\circ} \mathrm{C}$ to dissolve. After $90-100{ }^{\circ} \mathrm{C}$, a lithium hydroxide aqueous solution was added and saponified for 2 hours. Dehydrated at $130^{\circ} \mathrm{C}$ for $1 \mathrm{~h}$. The aqueous solution of lithium hydroxide and azelaic acid was added to carry out a reaction for 1 hour, and the mixture was heated to $160{ }^{\circ} \mathrm{C}$ for 1 hour, and then the remaining base oil was added, and the temperature was raised to $210{ }^{\circ} \mathrm{C}$ for 10 minutes. Final cooling, addition of additives and homogenization. The properties of the five samples are shown in Table 1. Samples 1 and 2 were commercial greases products, and the others were laboratory prepared samples.

Table 1. Basic Properties of Five Lithium Complex Grease

\begin{tabular}{|c|c|c|c|c|c|c|}
\hline Test items & sample1 & $\begin{array}{l}\text { sample } \\
2\end{array}$ & $\begin{array}{l}\text { sample } \\
3\end{array}$ & $\begin{array}{l}\text { sample } \\
4\end{array}$ & $\begin{array}{l}\text { sample } \\
5\end{array}$ & Test Methods \\
\hline Worked penetration $/ 0.1 \mathrm{~mm}$ & 279 & 232 & 213 & 256 & 225 & ISO 2137 \\
\hline $\begin{array}{l}\text { Prolonged worked penetration } \\
/ 0.1 \mathrm{~mm}\end{array}$ & 295 & 250 & 233 & 272 & 267 & ISO 2137 \\
\hline Dropping Point $/{ }^{\circ} \mathrm{C}$ & 304 & 275 & 317 & 316 & 315 & ISO 6299 \\
\hline oil separation $/ \%$ & 2.52 & 0.5 & 0 & 3.02 & 2.31 & $\begin{array}{l}\text { ASTM } \\
\text { D6184 }\end{array}$ \\
\hline
\end{tabular}

\section{Results and Discussion}

\subsection{Comparison of Microstructure}

The microstructures of the five lithium complex grease samples were compared, and the electron micrographs of the photographs are shown in Figure 1. It can be seen from the figure that the soap fiber network structure of the five samples has obvious differences. The soap fiber of sample 1 is slender, the diameter is the most uniform, and almost all the fibers exhibit a clear double helix-like structure, the whole fiber network is dense and interlaced. The fiber morphology and regularity of samples 2 and 3 were slightly worse than those of sample 1, and there were relatively coarse fibers. 
The soap fiber of sample 4 was uneven in diameter and length, and part of the soap fiber had no double helix structure. Sample 5 had the worst morphology of soap fibers, loose network structure, different diameter and length of soap fibers, and lumpy and flaky soap fibers. The difference in microstructure of the lithium complex grease sample will result in a change in macroscopic rheological properties.

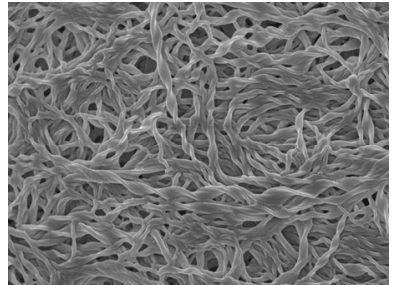

sample 1

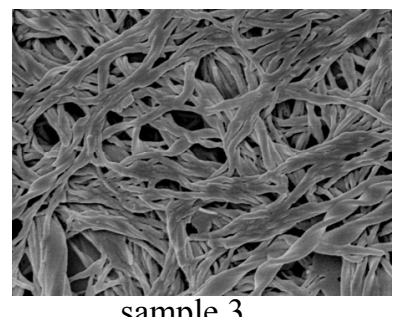

sample 3

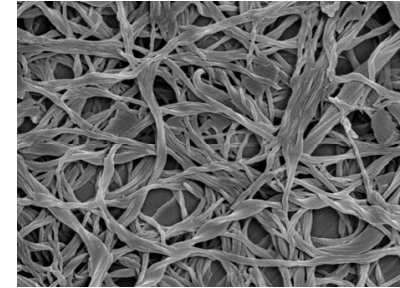

sample 2

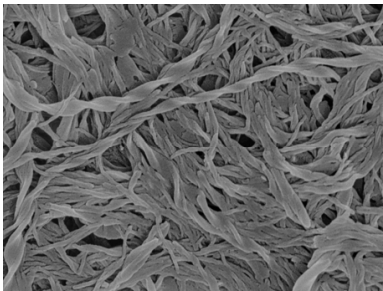

sample 4

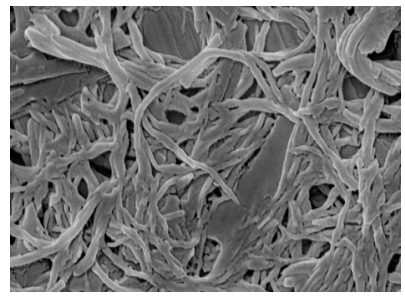

sample 5

Figure 1. SEM Photograph of Five Lithium Complex Greases

\subsection{Comparison of Viscoelasticity}

Grease is a semi-solid material that is both viscous and elastic. The oscillating experiments of five lithium complex grease samples were carried out by rheometer, and the relationship between the storage modulus G' and the loss modulus G" as the shear stress increased was obtained, as shown in Figure 2 and Figure 3, The storage modulus G' represents the elastic part of the viscoelasticity of the grease, indicating that the stress energy is temporarily stored in the test and can be recovered. The loss modulus $\mathrm{G}^{\prime}$ represents the viscous portion of the viscoelasticity of the grease, indicating that the energy used for the initial flow is an irreversible loss and the energy is converted to shear heat. At $25^{\circ} \mathrm{C}$ and $-40^{\circ} \mathrm{C}$, the modulus of the five samples decreased with the increase of stress. When the stress is small, G' is greater than G”, indicating that the samples have more similar structural features to solids. The point at which the storage modulus G' begins to decrease is usually defined as the end point of the linear viscoelastic region (LVE), which determines the maximum deformation that the sample can withstand before the 
internal structure is destroyed. The shear stress $\tau y$ at this time is also called yield point. In the linear viscoelastic region, the grease has gel characteristics, physical or chemical cross-linking network structure, G' and G" can characterize the structural strength and viscosity of the grease, and Thickener is the main factor affecting the structure of grease. It can be seen from Figure 2 that the modulus values of the five samples with higher consistency at normal temperature are also higher, and the corresponding yield stress $\tau y$ is also larger, which is consistent with the actual use of the grease. When the mechanical parts rotate, generally grease with a thicker consistency require a larger external force to deform, so the rotational torque is also higher. As the shear stress increases, the modulus decreases. When the shear stress $\tau>\tau y$, the sample enters the yielding zone. The soap fiber structure of the lithium complex grease begins to be destroyed, and the soap fibers begin to be rearranged, but at this time the sample still has gel characteristics, only partial deformation can be recovered; At higher shear stress, The lines G' and G" intersect at one point, also known as the flow point, the stress at $G^{\prime}=G$ ” is taken as the flow stress $\tau f$, indicating that the sample begins to flow at this time, and the fiber network structure of the lithium complex grease is greatly destructed, when the shear stress $\tau>\tau f$, the viscosity characteristic of the sample is greater than the elastic characteristic, G" $>\mathrm{G}$, indicating that the sample is in a liquid state and exhibits flow behavior.

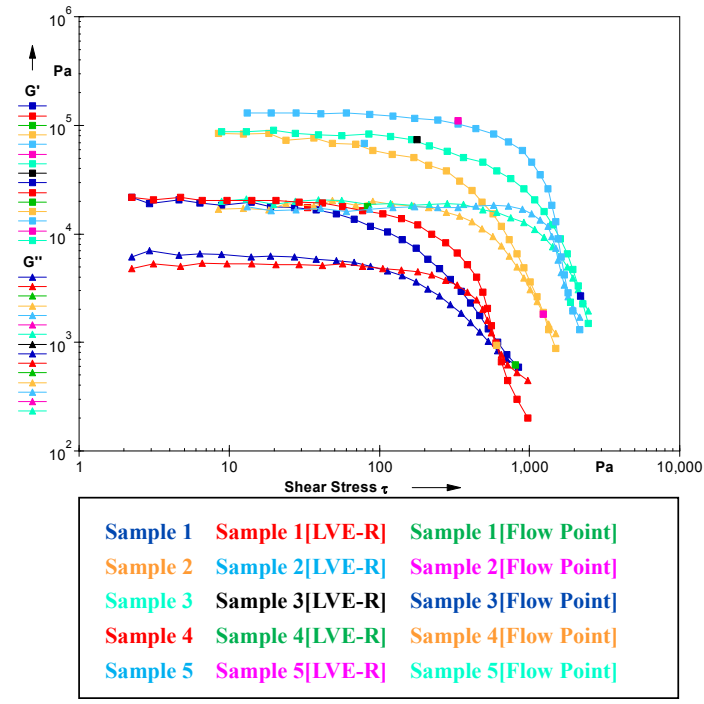

Figure 2. The Storage and Loss Modulus for Greases $\left(25^{\circ} \mathrm{C}\right)$ 


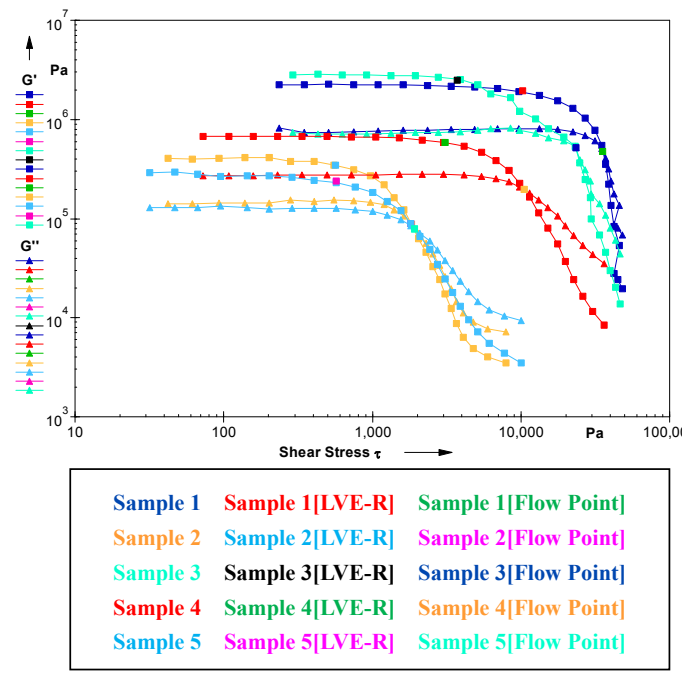

Figure 3. The Storage and Loss Modulus for Greases $\left(-40^{\circ} \mathrm{C}\right)$

The calculated viscoelastic characteristic parameters are shown in Table 2 and Table 3. The stress value $\tau f$ of the flow point is one of the important characteristics of the grease, and has significance in the application of the grease. The flow stress of the five samples at $-40^{\circ} \mathrm{C}$ was significantly increased compared with $25^{\circ} \mathrm{C}$, indicating that the fluidity of the lithium complex grease deteriorated at low temperatures. Comparing sample 2 and sample 3 with close consistency, it was found that the flow stress of sample 3 was much higher than that of sample 2 at $-40^{\circ} \mathrm{C}$, indicating that the low temperature fluidity of sample 2 was better. The flow transition index $\tau \mathrm{f} / \tau \mathrm{y}$ is also an important rheological characteristic parameter, which can describe the failure behavior of the internal structure of grease. The closer the value is to 1 , the more the grease exhibits brittleness, and the soap fibers are more susceptible to breakage when sheared. It can be seen from Table 2 that the $\tau \mathrm{f} / \tau \mathrm{y}$ of the five samples differs greatly at $25^{\circ} \mathrm{C}$, and the $\tau \mathrm{f} / \tau \mathrm{y}$ value of the sample 1 is the largest, indicating that the soap fiber structure is the most stable and tough, compared with the electron micrograph in Figure 1, The soap fiber of sample 1 has the most uniform morphology and the most compact network structure. The $\tau \mathrm{f} / \tau \mathrm{y}$ values of sample 2 and sample 3 are relatively close, and the morphology and uniformity of the soap fibers are similar in terms of microstructure. The $\tau \mathrm{f} / \tau \mathrm{y}$ value of sample 4 was much lower than that of sample 1 , and the corresponding microstructure was also significantly deteriorated, indicating that the soap fiber structure was more easily destroyed and the stability was poor when sheared by stress. The $\tau \mathrm{f} / \tau \mathrm{y}$ value of sample 5 was the smallest, and the grease structure was most easily destroyed. Compared with the microstructure, the soap fiber morphology of sample 5 was also the worst. Therefore, comparing the flow transition index $\tau \mathrm{f} / \tau \mathrm{r}$ of five samples at room temperature and the structure of soap fiber, it is found that there is a certain relationship, and the poorer the morphology of the soap fiber structure, the smaller the value of $\tau f / \tau y$. It can be seen from Table 3 that the grease will become hard and brittle at $-40^{\circ} \mathrm{C}$ due to the extremely low temperature, and the $\tau \mathrm{f} / \tau \mathrm{y}$ values of the five samples are significantly reduced and the difference is not large. It is indicated that the flow transition index $\tau \mathrm{f} / \tau \mathrm{y}$ of the lithium complex grease can be tested at room 
temperature, which can better reflect the stability of the internal structure of the sample. In addition, the loss factor or damping coefficient $\tan \delta=\mathrm{G} " / \mathrm{G}$ ' can also be used as an added value for grease performance description. The loss coefficient values in the linear viscoelastic region are shown in Table 2 and Table 3. The small loss factor value indicates that the sample is brittle and the loss factor is large, indicating that the sample is easier to spread. It can be seen from Table 2 that the sample has the largest $\tan \delta$ value at room temperature, and the soap fiber is finer and uniform in terms of microstructure, so that it is easier to spread and enter the raceway surface. The tan $\delta$ values of sample 2 , sample 3 , and sample 4 were substantially at one level. Sample 5 has the smallest $\tan \delta$ value, and its soap fiber structure is also the worst. The uneven soap fiber makes it difficult to enter the raceway, and the sample is brittle. At $-40^{\circ} \mathrm{C}$, the $\tan \delta$ values of the five samples increased to different extents, indicating that the brittleness of the grease became larger at low temperatures, but the relationship between the $\tan \delta$ value and the microstructure of the sample was not obvious, indicating the rheology of grease is not only related to thickeners, but also factors such as base oil at low temperature.

Table 2. Viscoelastic Characteristic Parameters for Lithium Complex Greases $\left(25^{\circ} \mathrm{C}\right)$

\begin{tabular}{llllll}
\hline \multirow{2}{*}{ Viscoelastic characteristic parameter } & \multicolumn{7}{l}{ Sample number } \\
\cline { 2 - 6 } & 1 & 2 & 3 & 4 & 5 \\
\hline Yield point $\tau \mathrm{y} / \mathrm{Pa}$ & 33.2 & 79.1 & 178 & 83.5 & 335 \\
Flow point $\tau \mathrm{f} / \mathrm{Pa}$ & 809.5 & 1238 & 2192 & 616.1 & 1873 \\
Flow transition index $\tau \mathrm{f} / \tau \mathrm{y}$ & 24.38 & 15.65 & 12.31 & 7.38 & 5.59 \\
Loss factor tan $\delta$ & 0.311 & 0.233 & 0.236 & 0.258 & 0.135 \\
\hline
\end{tabular}

Table 3. Viscoelastic Characteristic Parameters for Lithium Complex Greases $\left(-40^{\circ} \mathrm{C}\right)$

\begin{tabular}{llllll}
\hline \multirow{2}{*}{ Viscoelastic characteristic parameter } & \multicolumn{6}{l}{ Sample number } \\
\cline { 2 - 6 } & 1 & 2 & 3 & 4 & 5 \\
\hline Yield point $\tau \mathrm{y} / \mathrm{Pa}$ & 10200 & 562 & 3720 & 3070 & 571 \\
Flow point $\tau \mathrm{f} / \mathrm{Pa}$ & 35570 & 1814 & 23390 & 10470 & 1926 \\
Flow transition index $\tau \mathrm{f} / \tau \mathrm{y}$ & 3.49 & 3.23 & 6.29 & 3.41 & 3.37 \\
Loss factor $\tan \delta$ & 0.345 & 0.3745 & 0.2595 & 0.407 & 0.473 \\
\hline
\end{tabular}

\subsection{Comparison of Shear Viscosity}

Viscosity is a property of a material that undergoes flow and irreversible deformation under stress. The relationship between the shear stress $\tau$ of Newtonian fluid and the shear rate $\dot{\gamma}$ is $\tau=\eta \times \dot{\gamma}$. While the grease is a non-Newtonian fluid, the shear stress and shear rate are not proportional, so the shear viscosity $\eta$ can only be determined under the specified test conditions. The viscosity of the five lithium complex grease samples was measured at a constant shear rate as shown in Figure 4. 


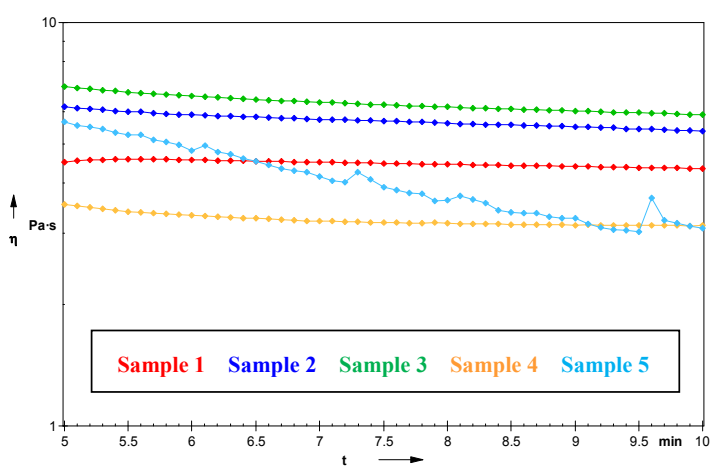

Figure 4. The Shear Viscosity Changes for Lithium Complex Greases

Since the grease is a shear-thinning fluid, the viscosity of the five samples decreased slightly under constant shear at 5 minutes. The initial viscosity $\eta_{A}$ and the final viscosity $\eta_{E}$ in the test period and the viscosity decrease rate $\eta_{r e l}$ are recorded from the graph, and are calculated according to the

equation $\eta_{r e l}=\frac{\eta_{A}-\eta_{E}}{\eta_{A}} \cdot 100$, expressed in units of percentage. As shown in Table 4, it can be seen that the viscosity drop rate of the sample 1 is the smallest, and a certain viscosity can be maintained after shearing, and the soap fiber structure is the most stable. The viscosity reduction rates of sample 2 , sample 3 , and sample 4 were substantially at one level, and the stability of the soap fiber structure was not much different. The viscosity of the sample 5 decreased the fastest, and the viscosity decreased by nearly half after 5 minutes of shearing, indicating that the shear resistance was poor. Therefore, the denser and more uniform the soap fiber structure of the lithium complex grease, the better the viscosity is maintained during the shearing process, and the poorer the soap fiber structure, the faster the viscosity decreases during shearing. If the viscosity drops too fast during use, it will be lost or smashed, which will eventually lead to lubrication failure.

Table 4. The Shear Viscosity for Lithium Complex Greases

\begin{tabular}{llllll}
\hline \multirow{2}{*}{ Shear viscosity } & \multicolumn{7}{l}{ Sample number } & & \\
\cline { 2 - 6 } & 1 & 2 & 3 & 4 & 5 \\
\hline Initial viscosity $\eta \mathrm{A} / \mathrm{Pa} \cdot \mathrm{s}$ & 4.54 & 6.14 & 6.90 & 3.51 & 5.57 \\
Final viscosity $\eta \mathrm{E} / \mathrm{Pa} \cdot \mathrm{s}$ & 4.35 & 5.38 & 5.91 & 3.14 & 3.09 \\
Relative viscosity drop rate $\eta \mathrm{rel} / \%$ & 4.17 & 12.3 & 14.3 & 10.5 & 44.5 \\
\hline
\end{tabular}

\section{Conclusion}

(1) By determining the rheological characteristic parameters of the grease, the stability and the degree of damage of the lithium complex grease structure can be described, which has certain guiding significance for the development and use of the product. The rheological properties of lithium complex greases are related to the microstructure of the grease and are the macroscopic representation of the internal 
microstructure of the grease. The lithium complex grease with excellent performance should have a dense and uniform, staggered three-dimensional network structure, and the soap fiber exhibits a distinct double helix structure.

(2) The viscoelastic characteristic parameters are related to the microstructure of the grease. The flow transition index $\tau \mathrm{f} / \tau \mathrm{y}$ is an important rheological characteristic parameter that can describe the failure behavior of the internal structure of the grease. The poorer the soap fiber structure of the lithium complex grease, the smaller the value of $\tau \mathrm{f} / \tau \mathrm{y}$, indicating that the soap fiber is more likely to break under the action of shearing force. The loss factor $\tan \delta$ can be used as an added value for grease performance description. The hourly loss factor indicates that the sample is brittle and the loss factor is large, indicating that the sample is easier to spread.

(3) At a constant shear rate, the denser and more uniform the soap fiber structure of the lithium complex grease, the better the viscosity is maintained during shearing, the smaller the relative viscosity drop rate, and the worse the soap fiber structure. The faster the viscosity decreases during the shearing process, the greater the rate of decrease in relative viscosity.

\section{References}

Delgado, M. A. et al. (2005). Relationship among Microstructure, Rheology and Processing of A Lithium Lubricating Grease. Chemical Engineering Research and Design, 83, 1085-1092. https://doi.org/10.1205/cherd.04311

DIN51810-1. (2007). Determination of the shear viscosity of greases using a rotary viscometer-Part 1: Cone-plate inspection system. Germany: German Standardization Association.

DIN51810-2. (2011). Determination of the rheological properties of greases-Part 2: Determination of the flow point using an oscillating rheometer with a parallel plate detection system. Germany: German Standardization Association.

Franco, J. M. et al. (2005). Mixing rheometry for studying the manufacture of lubricating greases. Chemical Engineering Science, 60, 2409-2418. https://doi.org/10.1016/j.ces.2004.10.042

Jiang, M. J. et al. (1998). Study on the rheological properties of grease: II. The flow of grease in the tube. Lubrication and Sealing, 23(4), 63-65.

Jiang, M. J., Guo, X. C., \& Jiang, B. X. (1997). Study on the rheological properties of semi-fluid fat. Synthetic Lubricating Materials, 2, 6-10.

Martin-Alfonso, J. E. et al. (2009). Influence of soap/polymer concentration ratio on the rheological properties of lithium lubricating greases modified with virgin LDPE. Journal of Industrial and Engineering Chemistry, 15, 687-693. https://doi.org/10.1016/j.jiec.2009.09.046

Meng, Y G., \& Zheng, J. (1998). A Rheological Model for Lithium Lubricating Grease. Tribology International, 31(10), 619-625. https://doi.org/10.1016/S0301-679X(98)00083-8

Wang, X. L., Zhu, T. B., \& Liang, H. F. (1997). Determination and research of rheological parameters of domestic grease. Journal of Tribology, 17(3), 232-237. 
Yeong, S. K., Luckham, P. F., \& Tadros, T .F. (2004). Steady flow and viscoelastic properties of lubricating grease containing various thickener concentrations. Journal of Colloid and Interface Science, 274, 285-293. https://doi.org/10.1016/j.jcis.2004.02.054 